\title{
Surgical treatment of slipped capital femoral epiphysis (SCFE) by Dunn procedure modified by Ganz: a systematic review
}

\author{
Giulio Gorgolini, Alessandro Caterini, Kristian Efremov, Lidio Petrungaro, Fernando De Maio, \\ Ernesto Ippolito and Pasquale Farsetti ${ }^{*}$ (D)
}

\begin{abstract}
Background: Treatment of SCFE is still controversial, especially in moderate and severe forms. Dunn osteotomy performed with the Ganz approach became very popular in the last decade, although it is a complicated and challenging surgical procedure with a risk of AVN. The aim of our study was to analyze the current literature verifying the effectiveness of this surgical procedure, with specific attention to the incidence of AVN and other complications.

Main body: A systematic review on the subject was performed according to the PRISMA guidelines. A literature search was performed by searching all published articles about the topic in the databases. The articles were screened for the presence of the following inclusion criteria: patients affected by slipped capital femoral epiphysis (SCFE) surgically treated by Dunn osteotomy using the Ganz surgical approach. All the patients affected by pathologies other than SCFE, treated without surgery or with procedures not including a surgical hip dislocation were excluded.

Based on inclusion and exclusion criteria, 23 studies were included in our systematic review. Selected articles were published from 2009 to 2021 and they included 636 overall hips. According to the selected articles, Dunn osteotomy modified by Ganz, performed by an experienced surgeon, allows for anatomical reduction of moderate or severe SCFE with a low incidence of AVN.

Conclusions: The few papers with long term follow-up, reported no progression of hip osteoarthritis, however, since the patients are adolescent at surgery, longer follow-up studies are needed to validate this statement. It is still debated if better results are obtained in stable or unstable SCFE. The indication of this procedure in mild SCFE remains controversial.
\end{abstract}

Level of evidence: 3

Keywords: Slipped capital femoral epiphysis, SCFE, Dunn osteotomy, Ganz surgical approach, Surgical hip dislocation, Flip trochanter osteotomy

*Correspondence: farsetti@uniroma2.it

Department of Clinical Sciences and Translational Medicine. Division of Orthopaedic Surgery, University of Rome "Tor Vergata", Viale Oxford 81, 00133 Rome, Italy

\begin{abstract}
Background
Slipped capital femoral epiphysis (SCFE) occurs with an overall incidence of 10.8 cases $/ 100.000$ children (1:7500 in males and 1:12.500 in females), between 9 and 16 years of age, with an average age of 13 years in males and 11.8, in females; it may be bilateral in 18 to $50 \%$ of the cases $[1,2]$. Its etiology remains uncertain, but histological, histochemical and ultrastructural
\end{abstract} original author(s) and the source, provide a link to the Creative Commons licence, and indicate if changes were made. The images or other third party material in this article are included in the article's Creative Commons licence, unless indicated otherwise in a credit line to the material. If material is not included in the article's Creative Commons licence and your intended use is not permitted by statutory regulation or exceeds the permitted use, you will need to obtain permission directly from the copyright holder. To view a copy of this licence, visit http://creativecommons.org/licenses/by/4.0/. The Creative Commons Public Domain Dedication waiver (http://creativeco mmons.org/publicdomain/zero/1.0/) applies to the data made available in this article, unless otherwise stated in a credit line to the data. 
changes have been reported in the pathogenesis of this disease $[3,4]$.

SCFE is commonly classified depending on the duration of the clinical symptoms in acute (up to 3 weeks), chronic (more than 3 weeks) and acute on chronic, and in stable and unstable based on the walking capacity of the affected patient $[5,6]$. In stable hips, the patient is able to bear weight with or without crutches, while in unstable hips, weight bearing is not possible even with crutches [6]. It is also classified from a radiographic point of view in three types, according to the severity of the posterior displacement of the capital epiphysis, which is measured using the Southwick angle; SCFE is defined mild when the Southwick angle measures $<30^{\circ}$, moderate when the angle measures between $30^{\circ}$ to $60^{\circ}$ and severe when it is $>60^{\circ}$ [7].

The classic treatment for acute or acute on chronic SCFE, generally unstable, was represented by an attempt of gentle closed reduction followed by a fixation in situ, while for the chronic forms, usually stable, the most common surgical treatment is represented by in situ pinning or by triplane trochanteric osteotomy, such as the Imhauser or Southwick osteotomy [7, 8]. Both these techniques, especially in moderate or severe forms, did not restore the anatomy of the femoral head and often femoro-acetabular impingement (FAI) occurs with premature hip osteoarthritis.

Dunn in 1964 first described a surgical technique for SCFE with the primary goal to "replace the femoral head on the end of the neck without stretching the retinacular vessels" [9]. In the original paper, he reported 19 good results out of 23 SCFE and four complications, two avascular necrosis (AVN) and two condrolysis. Several years later, Dunn and Angel better described the surgical technique that they performed in a cohort of 73 SCFE ( 25 acute on chronic and 48 severe chronic) [10]. They used a postero-lateral approach, performing the capsule incision along the axis of the femoral neck and extended it round the anterior and posterior edge of the acetabulum. The authors never dislocated the femoral head but with extreme care detached it from the femoral neck which was shortened; the head was anatomically reposition on the shortened neck and stabilized with three pins. The authors observed a clinicalradiological fair or poor result in $8-25 \%$ of the chronic slip and in $30-36 \%$ of acute on chronic slips and concluded that open reduction is an excellent treatment for severe chronic slipping in patients with open physis and that the main complications are observed in acute on chronic forms; they also speculated that the damage of the blood supply of the femoral head in these cases occurred at the time of the acute slip, before surgical reduction [10].
In 2001 Ganz et al. [11] first described a modification of the Dunn technique "with full access to the femoral head and acetabulum without risk of AVN". The operation consisted of an anterior dislocation of the hip based on detailed studies published by the same authors a year earlier on the vascular anatomy of the hip [12]. The authors, through a posterior/postero-lateral hip approach, performed a "trochanteric flip" osteotomy and a z-shaped capsulotomy anterior to the lesser trochanter, to preserve the deep branch of the medial femoral artery. They reported 213 surgical dislocations of the femoral head with various indications in patients with a mean age of 33.5 years, without a single case of AVN. The technique of surgical dislocation presented in their study allows a visualization of the entire femoral head and a complete access to the acetabulum, therefore in the subsequent years it was adopted by many orthopedic surgeons for approaching the hip joint in skeletally immature patients affected by SCFE.

The present study is a systematic review in which we analyzed all the available literature published after the description of the "surgical hip dislocation technique", reporting the clinical and radiological results with the incidence of AVN of the femoral head, in patients affected by SCFE surgically treated by Dunn osteotomy using the Ganz surgical approach. The aim of this study was to verify the effectiveness of this surgical procedure for the different types of SCFE, with specific attention to the incidence of AVN that in adolescent patients represents a dramatic complication only resolved through the application of a total hip prosthesis at a young age.

\section{Main text}

We performed a systematic review on the subject according to the PRISMA guidelines [13, 14]. Inclusion and exclusion criteria were formulated according to the PICO method [15] and they were summarized in Table 1.

Search strategy and sources of information:

Authors of this review (GG, AC, KE, FDM, PF) performed a literature search about the topic by querying Medline database. Studies were located by searching the databases. The search strategy covers PICO and was performed independently by each author in February 2021. Keywords and MeSH Terms were identified by a preliminary search and selected by discussion. The search was conducted using the following keywords and their synonyms, assembled in various combination to obtain the most pertinent articles: Slipped capital femoral epiphysis, SCFE, Dunn osteotomy, Ganz surgical approach, surgical hip dislocation.

The following search query were used: ((("slipped capital femoral epiphyses"[MeSH Terms] OR ("slipped"[All Fields] AND "capital"[All Fields] AND 
Table 1 Inclusion and exclusion criteria (PICO)

\begin{tabular}{|c|c|c|}
\hline & Inclusion criteria & Exclusion criteria \\
\hline Population & - Patients affected by slipped capital femoral epiphysis (SCFE) & - Patients who did not underwent surgery \\
\hline Intervention & - Dunn osteotomy modified by Ganz with surgical hip dislocation approach & $\begin{array}{l}\text { - Surgical techniques without hip dislocation } \\
\text { - Non-surgical treatment }\end{array}$ \\
\hline Comparison group & $\begin{array}{l}\text { - Studies reporting patients affected by SCFE treated by Dunn procedure } \\
\text { modified by Ganz, including comparative studies with in situ pinning or } \\
\text { Imhauser osteotomy }\end{array}$ & - Not applicable \\
\hline Outcome & - Studies reporting clinical and radiographic scores & - Not applicable \\
\hline Time & - Studies published from 2001 to 2021 & - Studies published prior to 2001 \\
\hline Study type & $\begin{array}{l}\text { - Clinical Trials } \\
\text { - Cohort Studies } \\
\text { - Observational Studies } \\
\text { - Randomized Control Trials }\end{array}$ & $\begin{array}{l}\text { - Letters } \\
\text { - Case reports } \\
\text { - Case series }<10 \text { hips }\end{array}$ \\
\hline Language & - English & - Other languages \\
\hline
\end{tabular}

"femoral"[All Fields $]$ AND "epiphyses"[All Fields]) OR "slipped capital femoral epiphyses"[All Fields] OR ("slipped"[All Fields] AND "capital"[All Fields] AND "femoral"[All Fields] AND "epiphysis"[All Fields]) OR "slipped capital femoral epiphysis"[All Fields]) OR ("Slipped Capital Femoral"[Title/Abstract]) OR ("Slipped Femoral"[Title/Abstract]) OR ("SCFE"[Title/ Abstract])) AND ((“GANZ”[Title/Abstract]) OR ("Dunn"[Title/Abstract]) OR ("Southwick"[Title/ Abstract]))) OR (("southwick"[All Fields] OR "southwick s"[All Fields]) AND ("osteotomie"[All Fields] OR "osteotomied"[All Fields] OR "osteotomy"[MeSH Terms] OR "osteotomy"[All Fields] OR "osteotomies"[All Fields])) OR (("ganz"[All Fields] AND ("osteotomie"[All Fields] OR "osteotomied"[All Fields] OR "osteotomy" [MeSH Terms] OR "osteotomy"[All Fields] OR "osteotomies"[All Fields]) AND (("slipped capital femoral epiphyses" [MeSH Terms] OR ("slipped"[All Fields] AND "capital"[All Fields] AND "femoral"[All Fields] AND "epiphyses"[All Fields]) OR "slipped capital femoral epiphyses"[All Fields] OR ("slipped"[All Fields] AND "capital"[All Fields] AND "femoral"[All Fields] AND "epiphysis"[All Fields]) OR "slipped capital femoral epiphysis"[All Fields]) OR ("Slipped Capital Femoral"[Title/Abstract]) OR ("Slipped Femoral"[Title/Abstract]) OR ("SCFE"[Title/Abstract])))) OR ("dunn"[All Fields] AND ("osteotomie"[All Fields] OR "osteotomied"[All Fields] OR "osteotomy"[MeSH Terms] OR "osteotomy"[All Fields] OR "osteotomies"[All Fields])) OR ("dunn procedure"[All Fields]) OR ("modified dunn osteotomy"[All Fields]).

Publication date filter was applied to select only articles from 2001 since it was the year in which Ganz et al. described for the first time their surgical approach for Dunn osteotomy. Language restriction were applied to identify only English articles.
The reviewers (GG, AC, KE, FDM, PF) retrieved the data and independently analyzed each selected study; instances of disagreement were resolved by the senior investigator (PF).

The articles were screened for the presence of the following inclusion criteria:

1) Patients affected by slipped capital femoral epiphysis (reporting at least 10 cases with a minimum followup of 1 year)

2) Patients surgically treated by Dunn osteotomy modified by Ganz with surgical hip dislocation technique

3) Studies with different level of evidence, including retrospective studies.

4) Availability of full text

5) Studies published from 2001 to 2021

The articles were excluded if any of the following exclusion criteria were identified:

1) Diagnostic or prognostic studies

2) Non-surgical treatment of patients

3) Other surgical approaches (in situ pinning, peritrochanteric osteotomy, Dunn osteotomy without hip dislocation)

4) Studies non pertinent with SCFE

5) Full text in a different language than English

6) Studies published prior to 2001

Figure 1 shows the flowchart for study selection.

The initial search produced 257 studies from Medline database, 18 studies from Scopus and 7 from WoS, for a total of 282 studies. Of the 25 studies found on Scopus and WoS, 19 were duplicates, while 6 were new unique entries. All records were screened by their title 
FLOW DIAGRAM OF SEARCH PROCESS

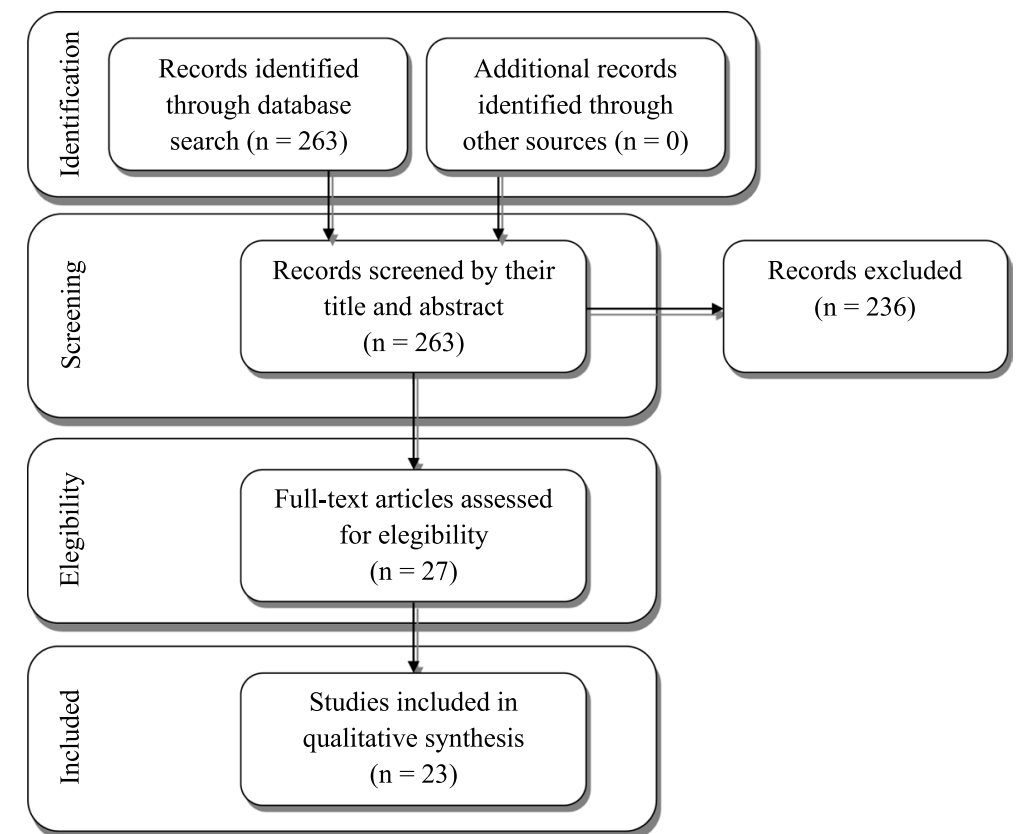

Fig. 1 Flow diagram, describing the number of studies identified, included and excluded with relative reasons

and abstract and 236 studies were excluded since they did not meet our inclusion criteria. They were excluded for the following reasons: 109 reported a not pertinent topic, 109 reporting a pertinent topic but did not meet all the inclusion criteria of the study design, 18 articles were published in a language different than English.

After detailed evaluation of the full text of the remaining 27 studies, based on inclusion and exclusion criteria, 23 papers were selected to be include in the present review [16-38]; 4 articles fulfilled the inclusion criteria but reported fewer than 10 cases.

Selected articles were published from 2009 to 2021 and they included 636 hips overall, with a follow up that ranged between 1.2 and 12 years. Of these, 399 were stable, while the remaining 237 unstable. Based on the onset, 174 hips were considered acute, 221 chronic, and 164 acute-on-chronic; 77 were not classified. Based on the slip angle, 29 hips were classified as mild $\left(<30^{\circ}\right), 97$ as moderate $\left(30^{\circ}-60^{\circ}\right)$ and 339 as severe $\left(60^{\circ}-90^{\circ}\right)$; one paper with 21 hips reported the mean slip angle of $59.1^{\circ}$, but not how many were in each category; this information was not available for the remaining 150 hips. The incidence of AVN was variable, with some studies reporting no cases of AVN, to some with a high incidence of up to $29.4 \%$ of AVN. A total of 69 cases of AVN were reported, with a mean incidence of $10.8 \%$. Other complications such as implant failure, heterotopic ossifications and limb length inequality were reported with various frequencies.
Of the 23 studies included in our review, most of them reported satisfactory results. Results were summarized and classified with validated scales and scores in 18 studies. One study reported results without a validated scale or score, while 4 studies did not report classified outcome values.

The most commonly used validated scales and scores reported to describe outcomes were Harris Hip Score (HHS) and The Merle d'Aubigné Hip Score (MdA), but in some papers results were reported using UCLA activity score, Hip Disability and Osteoarthritis Outcome Score (HOOS), Western Ontario and McMaster University score (WOMAC), Nottingham Health Profile score (NHP), Visual Analog Scale (VAS), and Heyman and Herdon score.

Fourteen studies reported an HHS with a mean value of 91,95 (range 76.3-99.6), 7 studies reported an MdA score with a mean value of $16.98,6$ studies reported a Womac score with a mean value of 96,2 . Three studies reported Heyman and Herdon excellent or good scores in 60, 67\% and $97,3 \%$ of cases respectively. Two studies reported a NAHS of 85.4 and 91.3. Finally, 1 last study reported satisfactory results in $94 \%$ of cases. Four studies did not report a classification of outcomes with outcome values.

Table 2 presents the list of reference of the studies, type of study, number of cases, average age at surgery, classifications, length of follow-up, results, incidence of AVN and other complications and conclusions. 


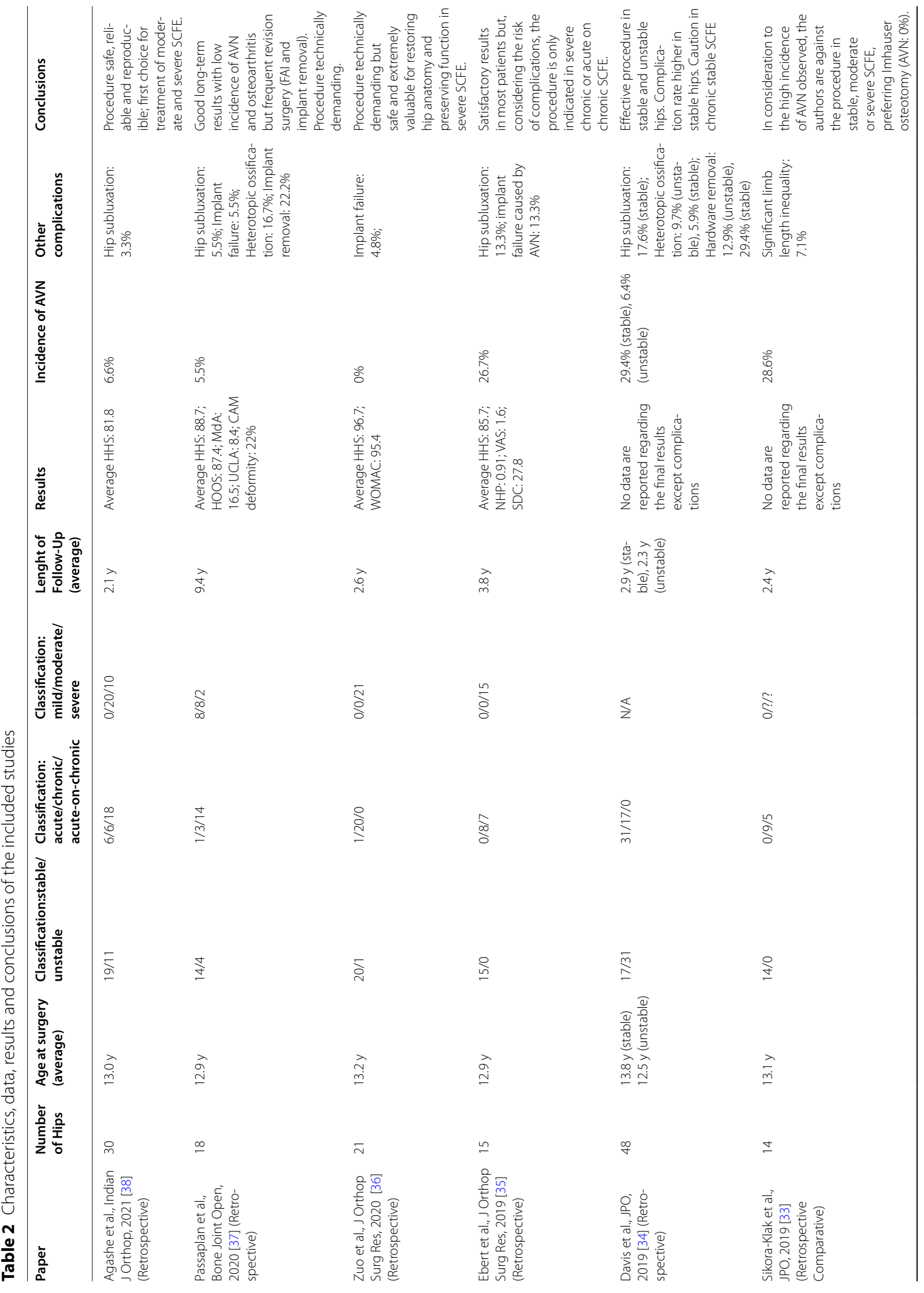




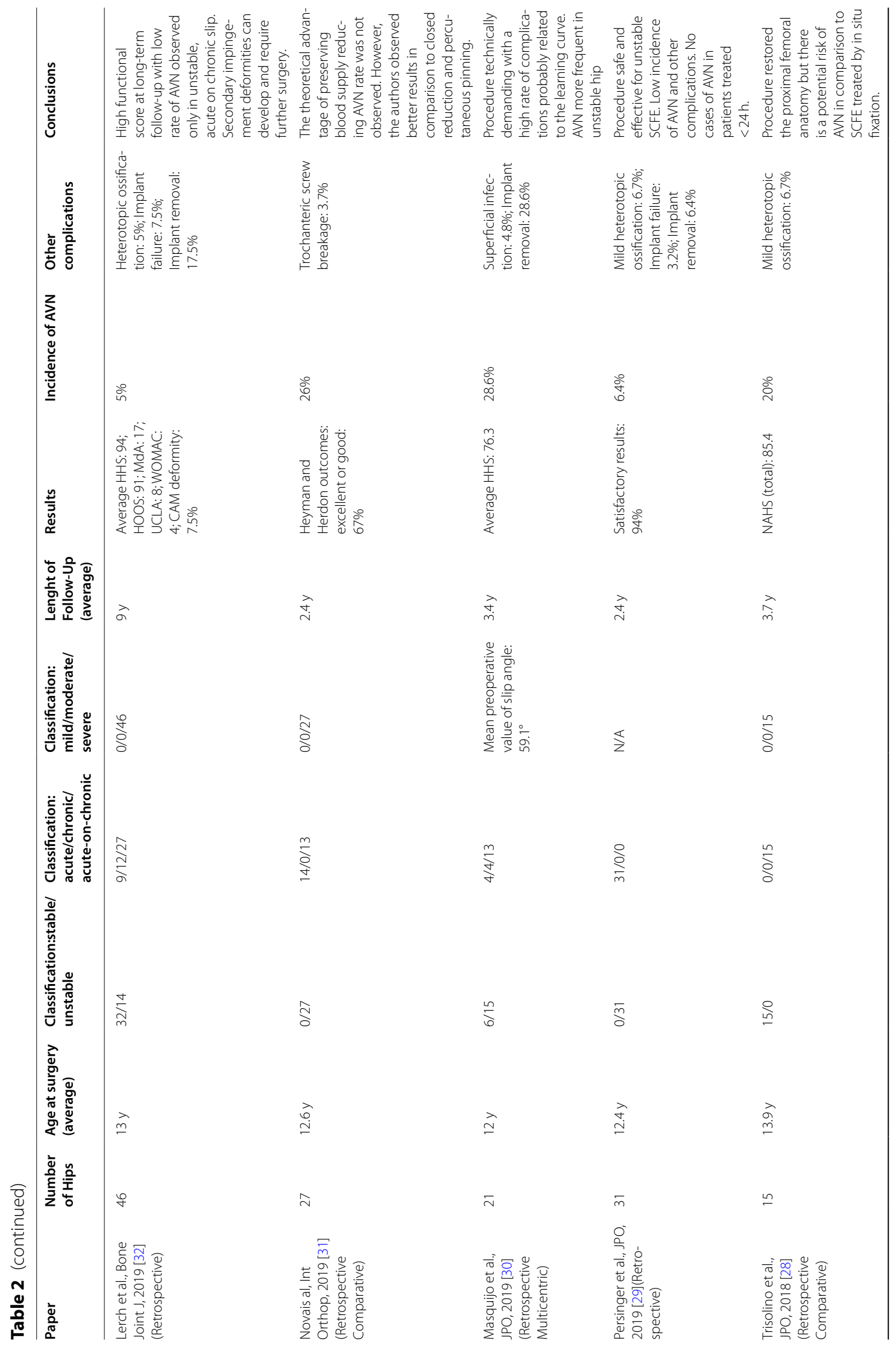




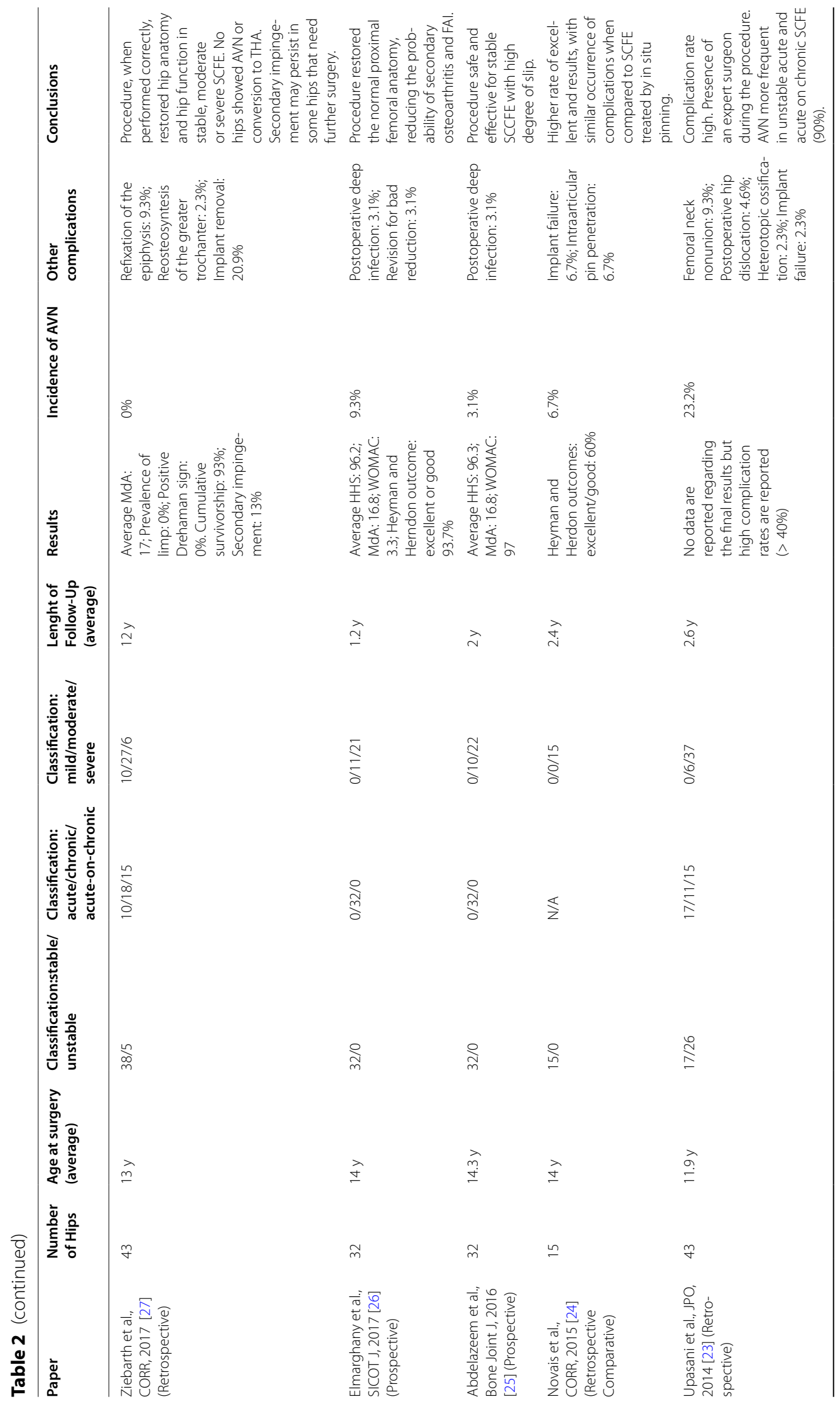




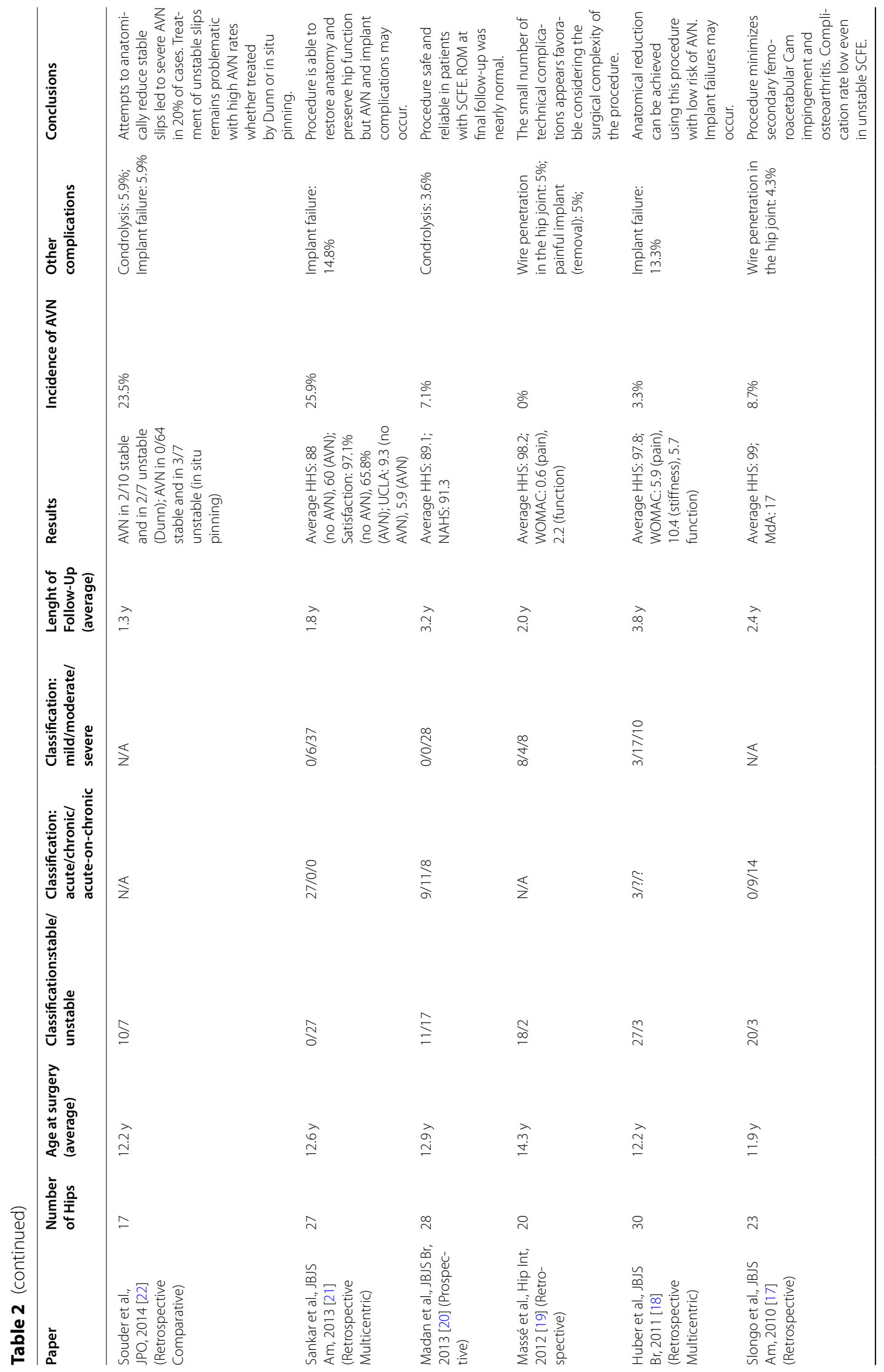




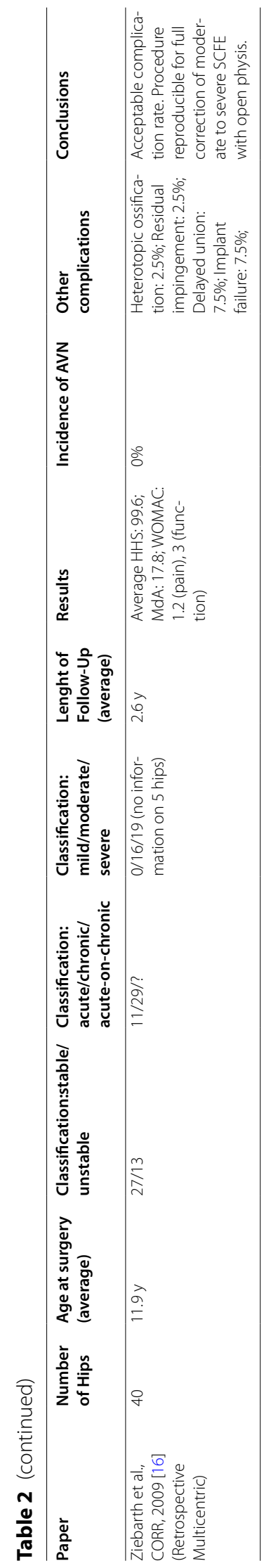


From the analysis of our selected literature, Dunn osteotomy modified by Ganz, performed by an experienced surgeon, allows for anatomical reduction of moderate or severe SCFE with a low incidence of AVN. In mild SCFE, there is no consensus on the use of this technique, since several authors prefer to perform an in situ fixation and treat possible FAI later on by hip arthroscopy.

The majority of the reported studies have a shortterm follow-up, which ranges from 1.2 to 3.8 years. Only three studies reported long term follow-up of 12, 9 and 9.4 years respectively. These studies seem to show the absence of degenerative hip osteoarthritis at follow-up in patients treated by this technique.

Treatment of SCFE is still controversial, especially in moderate and severe forms [39]. It is also related to the mode of onset of the disease, if acute or chronic or acute on chronic, and to the stability of the capital epiphysis on the femoral neck. According to our systematic review we believe that Dunn osteotomy modified by Ganz should be strongly considered in moderate and severe forms of SCFE, because it allows an anatomical reduction of the femoral head sparing vascular supply in the majority of the cases. There is no general consensus on using this technique in mild SCFE. The learning curve may be long and should be taken into consideration.

Generally, pinning in situ represents the treatment of choice for mild SCFE with the goal to prevent further slip progression [40], although some authors have reported mild forms treated by Dunn osteotomy modified by Ganz. In our series of selected papers, four studies reported an overall number of 29 patients affected by mild SCFE treated by osteotomy according to the Ganz surgical approach [18, 19, 27, 37]. Two studies, justify this option of treatment arguing that even mild forms of SCFE may cause secondary FAI which leads to late hip osteoarthritis $[19,27]$; both reported an overall number of 63 patients surgically treated with no cases of AVN. Huber et al. [18] suggested an intraoperative inspection of the slipping after the hip dislocation, before making the final decision whether to perform only in situ fixation or osteotomy. They reported three cases classified as mild SCFE in which the displacement was more evident than expected and needed a repositioning of the capital epiphysis. The last study that reported eight hips with a mild slip treated by osteotomy, reported in their limitations that all patients were surgically treated regardless of the severity, stability and chronicity of the slip [37]. We believe, in agreement with the majority of the authors, that, considering the risk of AVN that may still occur after surgery even in patients treated by expert hip surgeons, the preferred option of treatment of mild SCFE might be in situ fixation, treating in a second time possible FAI by arthroscopic trimming of the metaphysis [41].

On the contrary, in moderate and severe forms of SCFE, there is a high risk of degenerative joint disease caused by the consequent deformity of the femoral head. Therefore, a surgical correction by anatomical repositioning of the capital epiphysis on the femoral neck, should be the treatment of choice, even with an incidence of $A V N>20 \%$ [21-23, 28, 30, 31, 33-35]. Dunn osteotomy performed with Ganz approach represents the only surgical procedure for restoring the correct anatomy of the proximal part of the femur without stretching the retinaculum vessels, however the operation is still technically demanding. The majority of the studies in spite of a high rate of AVN, are in favor of this procedure, although there is disagreement regarding the indications in relation to the type of SCFE [21-23, 30, 31, 34, 35]. Davis et al. [34], reported better results in unstable hips in which the AVN rate was $6.4 \%$ in comparison to $29.4 \%$ observed in stable hips. On the contrary, other authors $[22,23]$ reported better results in stable SCFE with AVN more frequently observed in unstable acute and acute on chronic slips. Novais et al. [24], in a series of severe unstable hips, in spite of an incidence of $26 \%$ of AVN observed after Dunn procedure, reported better results in comparison to closed reduction and percutaneous pinning. Finally, some authors $[23,30]$ related their high incidence of AVN to the surgeon, stressing the importance of the learning curve and suggest the presence of an expert orthopaedist, reporting a statistically significant association between surgeon and incidence of complications [23].

The remaining 14 papers reported a low incidence of AVN, from 0 to $9.3 \%$. The total number of operated hips were 409, the Harris Hip Score ranged from 81.8 to 99.6 points, while the Merle d'Aubigné score ranged from 16.5 to 17.8 points. All these authors recommend Dunn procedure modified by Ganz in moderate and severe SCFE, because when it is performed correctly, it restored hip anatomy and function. They considered this procedure safe, reliable and reproducible, and recommend it as the first choice of treatment, although it is technically demanding and requires an expert surgeon. The higher incidence of AVN was observed in unstable acute on chronic SCFE [32].

Regarding the other complications, in the majority of the selected studies (17 papers) [16-19, 21-24, 27, 29$32,34-37]$ the authors performed implant removal for implant failure, related to AVN or different reasons. The incidence of implant removal ranged from 2.3 to $29.48 \%$. In five papers $[23,34,35,37,38]$ the authors observed hip instability with an incidence from 3.3 to $17.6 \%$ that 
required a second operation. This complication, as reported by some authors [42] may be directly related to SCFE such as damages of the acetabular labrum or the acetabular cartilage, causes not related to SCFE (acetabular orientation or poor quality of the soft tissues) and causes related to the surgical operation. They suggest to test "the congruity and stability of the hip during the surgical procedure and preferably treat this complication during the same period of anesthesia".

Heterotopic ossifications are also described in seven papers [16, 23, 28, 29, 32, 34, 37] with an incidence from 2.3 to $16.7 \%$, but in the majority of the cases they were completely asymptomatic. Other complications such as, deep infections [25, 26], femoral neck nonunion [23], condrolysis $[20,22]$ or significant limb length inequality [33] are extremely rare and reported by one or two papers with a low incidence.

Regarding the stability of the SCFE, many papers report their results of stable and unstable hips together, without differentiating them in terms of clinical results and complications, although the majority of the hips in these studies were stable (259 stable hips vs 121 unstable hips reported in 13 papers) [16-20, 22, 23, 27, 30, 32, 36-38]. Davies et al. [34], reported a cohort of 48 SCFE (17 stable and 31 unstable), differentiating their results according to the hip stability. They observed most frequent complications in stable hips (AVN: $29.4 \%$ vs $6.4 \%$, hip instability: $13.3 \%$ vs $0 \%$ ), but they concluded that, although the complications are higher in stable hips, the surgical procedure is effective in both groups. Six papers [24-26, 28, 33, 35] reported only patients with stable hips (123 hips), three of them had an incidence of $\mathrm{AVN}>20 \%$, while the other three, a low incidence. The remaining three papers $[21,29,31]$ reported only patients with unstable hips (85 hips), with a high incidence of AVN in two studies (about 26\%). These data confirm that Dunn osteotomy modified by Ganz is more frequently used in stable SCFE and the higher risk of AVN seems to be in unstable hips.

The majority of the reported studies have a shortterm follow-up, which ranges from 1.2 to 3.8 years. Only three studies reported long term follow-up of 12, 9 and 9.4 years respectively [27, 32, 37]. Ziebarth et al. [27] reported a 12 year long term follow-up study, analyzing 43 hips affected by SCFE operated according to modified Dunn procedure. The authors observed in $>90 \%$ of cases an excellent or good result with no progression of osteoarthritis. No hips showed signs of AVN at the latest follow-up. Only four hips showed progression of osteoarthritis between 10 and 17 years followup but no patient needed a total hip arthroplasty. No difference was found in survivorship between mild, moderate and severe slips. Lerch et al. [32] reported a
9 years follow-up retrospective study in 46 hips with severe SCFE treated by modified Dunn procedure. They reported a low risk of progression of hip osteoarthritis and an incidence of 5\% of AVN observed in two patients with an unstable acute on chronic slip that needed further surgery. FAI was observed in three patients, but only in one case the reduction was incomplete. The other two developed a later deformity related to the remodeling of the capital epiphysis. Passaplan et al. [37] reported a 9.4 year follow-up in which they analyzed 18 SCFE surgically treated by modified Dunn procedure. They reported a low rate of osteoarthritis and observed AVN only in two cases that were slightly symptomatic, with a good long-term clinical outcome. An asymptomatic osteoarthritis grade I was seen in both hips. Moreover, they observed five hips with an anterior impingement but only two of them had a pathological alfa angle on the axial view.

The majority of the papers are retrospective studies without a control group. Only five studies reported comparative results of Dunn osteotomy versus in situ pinning [22, 24, 28, 31] or Imhauser osteotomy [33]. There is general agreement that in severe stable hips, AVN is more frequent with modified Dunn osteotomy in comparison to pinning in situ (20\% vs $0 \%)$ [22, 24 , 28]. On the contrary, in unstable hips, similar or better results using modified Dunn procedure are reported [22, 31]. Only one paper compared Dunn procedure with Imhauser osteotomy; Sikora-Klak et al. [33] reported better results with Imhauser osteotomy in comparison to modified Dunn procedure. Some recent papers suggested to improve Imhauser osteotomy by performing a neck osteochondroplasty through a surgical dislocation approach. They considered this procedure safe and effective in 62 hips with severe stable SCFE after a mean follow-up of 4.1 years [43-45]. However, in a previous 24 years long term follow-up study in which SCFE were treated by Imhauser osteotomy, the incidence of osteoarthritis was high (45\%) [46].

In the few prospective studies $[20,25,26]$ the authors reported that Dunn osteotomy represents a safe and effective procedure that restored a normal proximal femoral anatomy with a nearly normal range of motion of the operated hip, reducing the probability of secondary osteoarthritis and FAI.

Based on our review, we propose a flow chart of surgical treatment according to clinical and radiographic classifications (Fig. 2).

There are some limitations with this systematic review. Most of the studies included on the Dunn osteotomy modified by Ganz are retrospective, and as such, the risk of selection and information bias is high. Long 


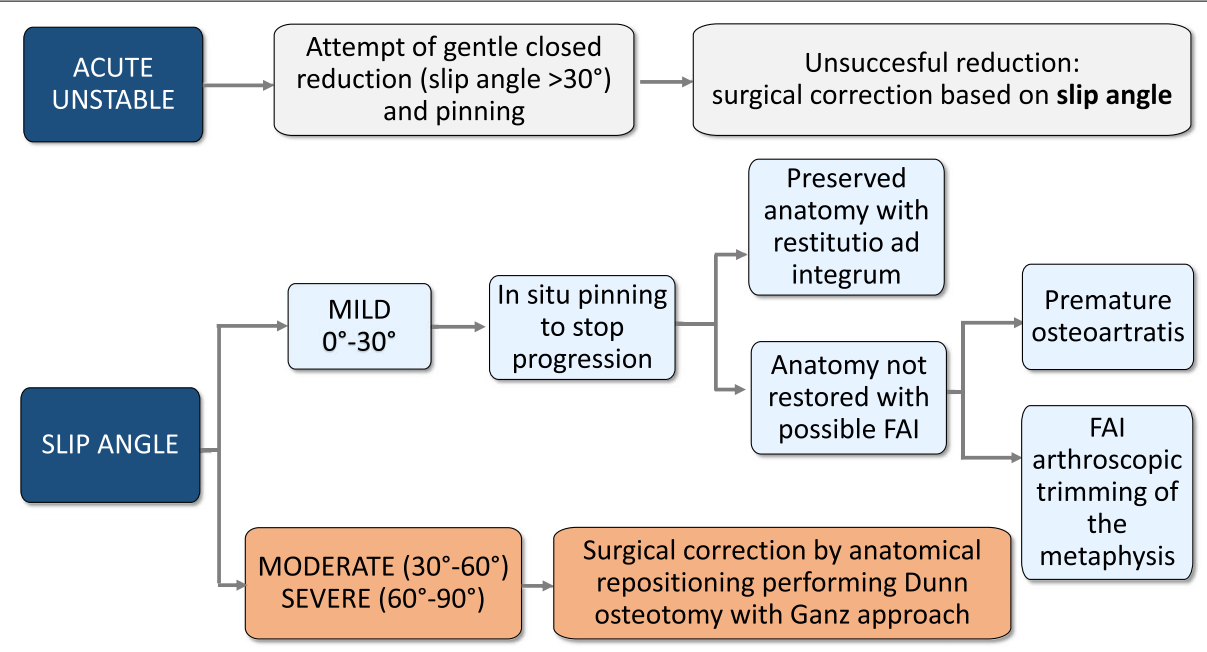

Fig. 2 Flow chart of surgical treatment according to clinical and radiographic classifications

term multicentric RCT are needed to better evaluate the efficacy of this treatment.

\section{Conclusions}

In conclusion, we believe that Dunn osteotomy modified by Ganz should be the preferred method to treat moderate or severe SCFE. The few papers with long term follow-up, reported no progression of hip osteoarthritis, however, since the patients are adolescents at surgery, more time is needed to evaluate the effectiveness of this treatment. It is still debated if better results are obtained in stable or unstable SCFE and further studies should focus on determining the importance of stability in the prognosis of this procedure. In mild SCFE, we believe that the possible risk of AVN of this procedure does not justify its use, since resulting FAI can be treated further down the line through hip arthroscopy.

\section{Abbreviations}

AVN: Avascular Necrosis; FAI: Femoro-Acetabular Impingement; PICO: Population Intervention Comparison Outcome; PRISMA: Preferred Reporting Items for Systematic Reviews and Meta-Analyses; RCT: Randomized Control Trial; SCFE: Slipped Capital Femoral Epiphysis.

\section{Acknowledgements}

Not applicable.

\section{About this supplement}

This article has been published as part of BMC Musculoskeletal Disorders Volume 22 Supplement 2 2021: All about the hip. The full contents of the supplement are available at https://bmcmusculoskeletdisord.biomedcentral.com/ articles/supplements/volume-22-supplement-2.

\section{Authors' contributions}

Conceptualization: PF, El. Data curation: GG, AC, KE, LP. Data analysis: GG, AC, KE, LP, FDM. Methodology: PF, GG, FDM. Project administration: PF. Validation: KE, El. Preparation of original draft: GG, PF. Writing, review and revision: KE. All authors read and approved the final manuscript.
Funding

There was no funding for this study. The publications costs were internally covered by the authors.

\section{Availability of data and materials}

The datasets used and/or analyzed in the current study are available from the corresponding author on reasonable request.

\section{Declarations}

Ethics approval and consent to participate

Not applicable.

\section{Consent for publication}

Not applicable.

\section{Competing interests}

The authors declare that they have no competing interests.

Received: 26 January 2022 Accepted: 27 January 2022

Published online: 07 February 2022

\section{References}

1. Lehmann CL, Arons RR, Loder RT, Vitale MG. The epidemiology of slipped capital femoral epiphysis: an update. J Pediatr Orthop. 2006;26:286-90.

2. Herngren B, Stenmarker M, Vavruch L, Hagglund G. Slipped capital femoral epiphysis: a population-based study. BMC Musculoskelet Disord. 2017; 18:304.

3. Ippolito E, Bellocci M, Farsetti P, Tudisco C, Perugia D. An ultrastructural study of slipped capital femoral epiphysis: pathogenetic considerations. J Orthop Res. 1989;7:252-9.

4. Tresoldi I, Modesti A, Dragoni M, Potenza V, Ippolito E. Histological, histochemical and ultrastructural study of slipped capital femoral epiphysis. J Child Ortop. 2017:11(2):87-92.

5. Fahey JJ, O'Brien ET. Acute slipped capital femoral epiphysis: review of the literature and report of ten cases. J Bone Joint Surg. 1965;47A:1105-27.

6. Loder RT, Richards BS, Shapiro PS, Reznick LR, Aronson DD. Acute slipped capital femoral epiphysis: the importance of physeal stability. J Bone Joint Surg. 1993;75A:1134-40.

7. Southwick WO. Osteotomy through the lesser trochanter for slipped capital femoral epiphysis. J Bone Joint Surg. 1967;49A:807-35. 
8. Imhauser G. Die Imhauser-osteotomie bei floriden gleitprozess. Bemerkungen zu der gleichlautenden Arbeit von Weber BG. Z Orthop Ihre Grenzgeb. 1966;102:327-9.

9. Dunn DM. The treatment of adolescent slipping of the upper femoral epiphysis. J Bone Joint Surg. 1964;46B:621-9.

10. Dunn DM, Angel JC. Replacement of the femoral head by open operation in severe adolescent slipping of the upper femoral epiphysis. J Bone Joint Surg. 1978;60B:394-403.

11. Ganz R, Gill TJ, Gautier E, Ganz K, Krugel N, Berlemann U. Surgical dislocation of the adult hip. J Bone Joint Surg. 2001;83B:1119-24.

12. Gautier E, Ganz K, Krugel N, Gill TJ, Ganz R. Anatomy of the medial femoral circumflex artery and its surgical implications. J Bone Joint Surg. 2000;82B:679-83.

13. PRISMA. PRISMA - transparent reporting of systematic reviews and Metaanalyses. PRISMA 2015.

14. Liberati A, Altman DG, Tetzlaff J, et al. The PRISMA statement for reporting systematic reviews and meta-analyses of studies that evaluate healthcare interven-tions: explanation and elaboration. BMJ. 2009;339:b2700.

15. Riva JJ, Malik KM, Burnie SJ, Endicott AR, Busse JW. What is your research question? An introduction to the PICOT format for clinicians. J Can Chiropr Assoc. 2012;56:167-71.

16. Ziebarth K, Zilkens C, Spencer S, Leunig M, Ganz R, Kim YJ. Capital realignment for moderate and severe SCFE using a modified Dunn procedure. Clin Orthop Relat Res. 2009;467(3):704-16.

17. Slongo T, Kakaty D, Krause F, Ziebarth K. Treatment of slipped capital femoral epiphysis with a modified Dunn procedure. J Bone Joint Surg Am. 2010;92(18):2898-908.

18. Huber H, Dora C, Ramseier LE, Buck F, Dierauer S. Adolescent slipped capital femoral epiphysis treated by a modified Dunn osteotomy with surgical hip dislocation. J Bone Joint Surg Br. 2011;93(6):833-8.

19. Massè A, Aprato A, Grappiolo G, Turchetto L, Campacci A, Ganz R. Surgical hip dislocation for anatomic reorientation of slipped capital femoral epiphysis: preliminary results. Hip Int. 2012;22(2):137-44.

20. Madan SS, Cooper AP, Davies AG, Fernandes JA. The treatment of severe slipped capital femoral epiphysis via the Ganz surgical dislocation and anatomical reduction: a prospective study. Bone Joint J. 2013;95-b(3):424-9.

21. Sankar WN, Vanderhave KL, Matheney T, Herrera-Soto JA, Karlen JW. The modified Dunn procedure for unstable slipped capital femoral epiphysis: a multicenter perspective. J Bone Joint Surg Am. 2013;95(7):585-91.

22. Souder CD, Bomar JD, Wenger DR. The role of capital realignment versus in situ stabilization for the treatment of slipped capital femoral epiphysis. J Pediatr Orthop. 2014;34(8):791-8.

23. Upasani W, Matheney TH, Spencer SA, Kim YJ, Millis MB, Kasser JR. Complications after modified Dunn osteotomy for the treatment of adolescent slipped capital femoral epiphysis. J Pediatr Orthop. 2014;34(7):661-7.

24. Novais EN, Hill MK, Carry PM, Heare TC, Sink EL. Modified Dunn procedure is superior to in situ pinning for short-term clinical and radiographic improvement in severe stable SCFE. Clin Orthop Relat Res. 2015;473(6):2108-17.

25. Abdelazeem AH, Beder FK, Abdel Karim MM, Abdelazeem H, Abdel-Ghani $H$. The anatomical reduction of a moderate or severe stable slipped capital femoral epiphysis by modified Dunn subcapital osteotomy using the Ganz approach: functional and radiological outcomes. Bone Joint J. 2016;98-b(9):1283-8.

26. Elmarghany M, Abd El-Ghaffar TM, Seddik M, Akar A, Gad Y, Ragheb E, et al. Surgical hip dislocation in treatment of slipped capital femoral epiphysis. Sicot j. 2017;3:10.

27. Ziebarth K, Milosevic M, Lerch TD, Steppacher SD, Slongo T, Siebenrock KA. High survivorship and little osteoarthritis at 10-year Followup in SCFE patients treated with a modified Dunn procedure. Clin Orthop Relat Res. 2017:475(4):1212-28

28. Trisolino G, Stilli S, Gallone G, Santos Leite P, Pignatti G. Comparison between modified Dunn procedure and in situ fixation for severe stable slipped capital femoral epiphysis. Acta Orthop. 2018;89(2):211-6.

29. Persinger F, Davis RL 2nd, Samora WP, Klingele KE. Treatment of unstable slipped capital epiphysis via the modified Dunn procedure. J Pediatr Orthop. 2018;38(1):3-8.

30. Masquijo JJ, Allende V, D’Elia M, Miranda G, Fernández CA. Treatment of slipped capital femoral epiphysis with the modified Dunn procedure: a multicenter study. J Pediatr Orthop. 2019;39(2):71-5.
31. Novais EN, Maranho DA, Heare T, Sink E, Carry PM, O'Donnel C. The modified Dunn procedure provides superior short-term outcomes in the treatment of the unstable slipped capital femoral epiphysis as compared to the inadvertent closed reduction and percutaneous pinning: a comparative clinical study. Int Orthop. 2019;43(3):669-75.

32. Lerch TD, Vuilleumier S, Schmaranzer F, Ziebarth K, Steppacher SD, Tannast M, et al. Patients with severe slipped capital femoral epiphysis treated by the modified Dunn procedure have low rates of avascular necrosis, good outcomes, and little osteoarthritis at long-term follow-up. Bone Joint J. 2019;101-b(4):403-14.

33. Sikora-Klak J, Bomar JD, Paik CN, Wenger DR, Upasani V. Comparison of surgical outcomes between a Triplane proximal femoral osteotomy and the modified Dunn procedure for stable, moderate to severe slipped capital femoral epiphysis. J Pediatr Orthop. 2019;39(7):339-46.

34. Davis RL 2nd, Samora WP 3rd, Persinger F, Klingele KE. Treatment of unstable versus stable slipped capital femoral epiphysis using the modified Dunn procedure. J Pediatr Orthop. 2019;39(8):411-5.

35. Ebert N, Rupprecht M, Stuecker R, Breyer S, Stiel N, Priemel MH, et al. Outcome of the modified Dunn procedure in severe chronic or acute on chronic slipped capital femoral epiphysis. J Orthop Surg Res. 2019;14(1):349

36. Zuo B, Zhu JF, Wang XY, Wang CL, Ma F, Chen XD. Outcome of the modified Dunn procedure in severe slipped capital femoral epiphysis. J Orthop Surg Res. 2020;15(1):506.

37. Passaplan C, Gautier L, Gautier E. Long-term follow-up of patients undergoing the modified Dunn procedure for slipped capital femoral epiphysis. Bone Jt Open. 2020;1(4):80-7.

38. Agashe MV, Pinto DA, Vaidya S. Modified Dunn osteotomy for moderate and severe slipped capital femoral epiphysis - a retrospective study of thirty hips. Indian J Orthop. 2021;55:100-8.

39. Johari AN, Pandey RA. Controversies in management of slipped capital femoral epiphysis. World J Orthop. 2016;7:78-81.

40. Libor LM, Sink EL. Risks and benefits of the modified Dunn approach for treatment of moderate or severe slipped capital femoral epiphysis. J Pedistr Orthop. 2013;33(suppl. 1):S99-S102.

41. Oduwole KO, de Sa D, Kay J, Findakli F, Duong A, Simunovic N, et al. Surgical treatment of femoroacetabular impingement following slipped capital femoral epiphysis: a systematic review. Bone Joint Res. 2017;6:472-80.

42. Aprato A, Leunig M, Massé A, Slongo T, Ganz R. Instability of the hip after anatomical re-alignment in patients with a slipped capital femoral epiphysis. Bone Joint J. 2017;99B:16-21.

43. Erickson JB, Samora WP, Klingele KE. Treatment of chronic, stable slipped capital femoral epiphysis via surgical hip dislocation with combined osteochondroplasty and Imhauser osteotomy. J Child Orthop. 2017;11:284-8.

44. Baraka MM, Hefny HM, Thakeb MF, Fayyad TA, Abdelazim H, Hefny MH, et al. Combined Imhauser osteotomy and osteochondroplasty in slipped capital femoral epiphysis through surgical hip dislocation approach. J Child Orthop. 2020;14:190-200.

45. Abdelaziz TH, Elbeshry SS, Goda AH, Fayyad TA, Aly AS, Mahmoud SA. Intertrochanteric Imhäuser osteotomy combined with osteochondroplasty in treatment of moderate-severe stable slipped capital femoral epiphysis: a case series study. J Pediatr Orthop B. 2020;29:283-91.

46. Schai PA, Exner GU. Corrective Imhäuser intertrochanteric osteotomy. Oper Orthop Traumatol. 2007;19:368-88.

\section{Publisher's Note}

Springer Nature remains neutral with regard to jurisdictional claims in published maps and institutional affiliations. 